\title{
Insight into 3-node triangular shell finite elements: the effects of element isotropy and mesh patterns
}

\author{
Phill-Seung Lee ${ }^{\mathrm{a}}$, Hyuk-Chun Noh ${ }^{\mathrm{b}}$, Klaus-Jürgen Bathe ${ }^{\mathrm{c}, *}$ \\ a Samsung Heavy Industries, 825-13 Yeoksam, Gangnam, Seoul 135-080, Korea \\ ${ }^{\mathrm{b}}$ Korea Concrete Institute Research Center, 635-4 Yeoksam, Gangnam, Seoul 135-703, Korea \\ ${ }^{\mathrm{c}}$ Department of Mechanical Engineering, Massachusetts Institute of Technology, 77 Massachusetts Avenue, Cambridge, MA 02139, USA
}

Received 9 October 2006; accepted 30 October 2006

Available online 28 December 2006

\begin{abstract}
In this paper, we study the convergence characteristics of some 3-node triangular shell finite elements. We review the formulations of three different isotropic 3-node elements and one non-isotropic 3-node element. We analyze a clamped plate problem and a hyperboloid shell problem using various mesh topologies and present the convergence curves using the s-norm. Considering simple bending tests, we also study the transverse shear strain fields of the shell finite elements. The results and insight given are valuable for the proper use and the further development of triangular shell finite elements.
\end{abstract}

(C) 2006 Elsevier Ltd. All rights reserved.

Keywords: Shell structures; Finite elements; Triangular elements; MITC elements

\section{Introduction}

For several decades, the finite element method has been used as a main tool to analyze shell structures in various engineering applications. However, there are still many important research challenges to increase the effectiveness of the analysis of shells [1-4].

Shells are three-dimensional structures with one dimension, the thickness, small compared to the other two dimensions. As the shell thickness decreases, shell structures can behave differently depending on the geometry, loading and boundary conditions of the shell, that is, the behavior of a shell structure belongs to one of three different asymptotic categories: membrane-dominated, bendingdominated, or mixed shell problems [2-4].

A major difficulty in the development of shell finite elements is to overcome the locking phenomenon for bendingdominated shells. When the finite element approximations

\footnotetext{
* Corresponding author. Tel.: +1 617253 6645; fax: +1 6172532275 .

E-mail address: kjb@mit.edu (K.J. Bathe).
}

cannot sufficiently well approximate the pure bending displacement fields, membrane and shear locking occur. Then, as the shell thickness decreases, the convergence of the finite element solution rapidly deteriorates. An ideal finite element formulation would uniformly converge to the exact solution of the mathematical model irrespective of the shell geometry, asymptotic category and thickness. In addition, the convergence rate should be optimal. Of course, it is extremely hard to reach ideal (or uniformly optimal) shell finite elements but continuous efforts are highly desirable.

When modeling general engineering structures, some triangular finite elements are frequently used. Typically, to mesh complex shell structures, the mesh generation scheme establishes by far mostly quadrilateral elements but when these become too distorted because of geometric complexities, triangular elements are used instead. Also, triangular shell elements may be effective when these are used to represent a thin structure within tetrahedral three-dimensional element meshes, like in the analysis of rubber media reinforced by thin steel layers, or in the solution of fluid-structure interactions [5]. Of course, in general, quadrilateral 
elements can have a higher predictive capability, and therefore more research effort has been expended to develop quadrilateral shell finite element discretizations and more progress has also been achieved. Since quadrilateral elements have simpler coordinate systems and richer strain fields than triangular elements, quadrilateral shell finite elements that overcome the locking phenomenon are also easier to establish. Indeed, some quadrilateral shell elements are close to "uniformly optimal" [1,2].

The two basic approaches used to formulate general shell elements $[1,2,6]$ are the formulations in which plate bending and membrane actions are superimposed and the formulations based on three-dimensional continuum mechanics $[1,2,7]$. However, discretizations based on elements in which the membrane and bending actions are superimposed may not converge in the solution of general shell problems [2], and we focus in our work on a general continuum mechanics based approach. The resulting elements are attractive because they can be used for any shell geometry and, also, a linear formulation can directly and elegantly be extended to general nonlinear formulations. However, the elements need be developed in mixed formulations, since the pure displacement formulation locks $[1,2,8]$. In particular, the displacement-based 3-node triangular shell finite element (QUAD3) locks severely. One approach is to use selective reduced integration resulting in the SRI3 element.

Recently, using the MITC $^{1}$ (Mixed Interpolation of Tensorial Components) technique for triangular shell finite elements, a 3-node MITC triangular shell finite element (MITC3) has been developed [9]. Its performance has been studied for various shell problems using well-established benchmark procedures. The element is very attractive because its formulation is simple and general, and, in particular, the behavior of the element is isotropic, that is, the stiffness matrix of the triangular element does not depend on the sequence of node numbering. However, the element is not "uniformly optimal", that is, some locking is present and seen in the solution of the clamped plate problem and the hyperboloid shell problem [9]. This deficiency provides a motivation to further study the element behavior.

It is well known that triangular shell finite elements give very different solution accuracy depending on the mesh pattern used for a shell problem $[1,10]$. Hence, to evaluate a triangular shell finite element, specific different meshes should be used to test the element performance. In addition also an appropriate norm need be used to measure the error [11].

Our objective in this paper is to further study the convergence behavior of the MITC3 shell finite element and some other 3-node triangular shell finite elements when using different mesh patterns and the s-norm proposed by Hiller and Bathe [11]. Also, to obtain insight into the reasons why the different results are obtained, we study

\footnotetext{
${ }^{1}$ The MITC technique has been successfully used for developing highperformance quadrilateral shell finite elements, namely the MITC4, MITC9 and MITC16 elements.
}

the transverse shear strain fields of the 3-node shell finite elements in simple bending problems.

While we use exclusively 3-node triangular shell finite elements in these studies, we recognize that - as pointed out above already - in practice these elements will frequently not be used alone but only when necessary together with quadrilateral elements. However, this fact does not diminish the importance of our study.

In the following sections, we first review the formulations of four 3-node triangular shell finite elements and their strain fields. Next, considering a fully clamped plate problem and a hyperboloid shell problem, we study the convergence of the shell finite elements depending on the mesh patterns used. To further investigate the behavior of the shell finite elements, we then study the transverse shear strain fields in two simple plate bending problems. Since the s-norm is used in the convergence studies, we give in an Appendix, a general scheme for the numerical calculation of this norm.

\section{Formulations of 3-node triangular shell finite elements}

We briefly review the formulations of four different 3node triangular shell finite elements: three isotropic elements and one non-isotropic element. Here, we only show the covariant strain fields of the elements since, once these fields are known, it is straightforward to establish the stiffness matrices for the analysis of shell structures [1].

\subsection{Covariant strain fields of 3-node triangular shell finite elements}

The geometry of a $q$-node continuum mechanics based shell finite element is described by

$\vec{x}(r, s, \xi)=\sum_{i=1}^{q} h_{i}(r, s) \vec{x}_{i}+\frac{\xi}{2} \sum_{i=1}^{q} t_{i} h_{i}(r, s) \vec{V}_{n}^{i}$,

where $h_{i}(r, s)$ is the 2D shape function of the standard isoparametric procedure corresponding to node $i, \vec{x}_{i}$ is the position vector for node $i$ in the global Cartesian coordinate system, and $t_{i}$ and $\vec{V}_{n}^{i}$ denote the shell thickness and the director vector at node $i$, respectively (see Fig. 1).

From Eq. (1), the displacement of the element is given by

$\vec{u}(r, s, \xi)=\sum_{i=1}^{q} h_{i}(r, s) \vec{u}_{i}+\frac{\xi}{2} \sum_{i=1}^{q} t_{i} h_{i}(r, s)\left(-\vec{V}_{2}^{i} \alpha_{i}+\vec{V}_{1}^{i} \beta_{i}\right)$,

in which $\vec{u}_{i}$ is the nodal displacement vector in the global Cartesian coordinate system, $\vec{V}_{1}^{i}$ and $\vec{V}_{2}^{i}$ are unit vectors orthogonal to $\vec{V}_{n}^{i}$ and to each other, and $\alpha_{i}$ and $\beta_{i}$ are the rotations of the director vector $\vec{V}_{n}^{i}$ about $\vec{V}_{1}^{i}$ and $\vec{V}_{2}^{i}$ at node $i$.

For a 3-node triangular shell finite element, $q$ is 3 and the shape functions are

$h_{1}=1-r-s, \quad h_{2}=r, \quad h_{3}=s$. 


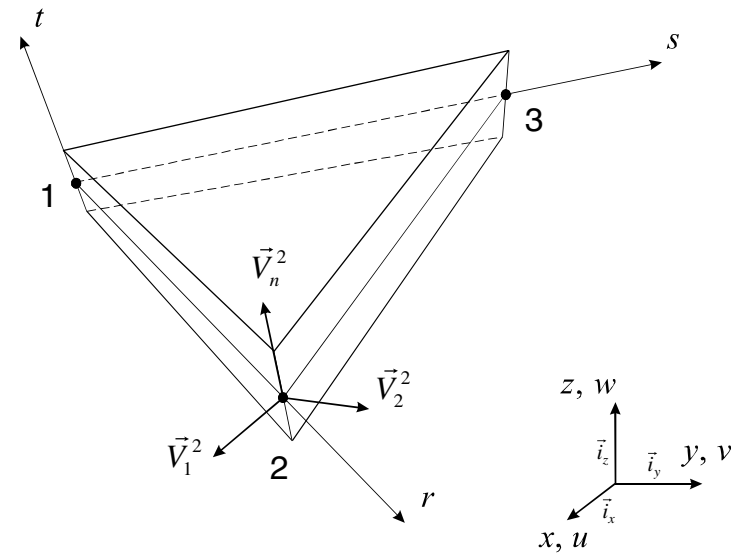

Fig. 1. A 3-node triangular continuum mechanics based shell finite element.

The linear part of the covariant strain components are directly calculated by

$e_{i j}=\frac{1}{2}\left(\vec{g}_{i} \cdot \vec{u}_{, j}+\vec{g}_{j} \cdot \vec{u}_{i}\right)$,

where

$\vec{g}_{i}=\frac{\partial \vec{x}}{\partial r_{i}}, \quad \vec{u}_{,_{i}}=\frac{\partial \vec{u}}{\partial r_{i}} \quad$ with $r_{1}=r, r_{2}=s, r_{3}=\xi$.

All 3-node shell elements considered here are flat, and the in-plane strain components are directly calculated using Eq. (4). However, the transverse shear strains are evaluated differently for each element as we next summarize.

- The QUAD3 element.

The covariant transverse shear strain field of the original displacement-based 3-node triangular shell finite element is directly calculated by Eqs. (1), (2) and (4) as follows:

$e_{r \xi}=\frac{1}{2}\left(\vec{g}_{r} \cdot \vec{u}_{,_{\xi}}+\vec{g}_{\xi} \cdot \vec{u}_{r}\right), \quad e_{s \xi}=\frac{1}{2}\left(\vec{g}_{s} \cdot \vec{u}_{,_{\xi}}+\vec{g}_{\xi} \cdot \vec{u}_{s}\right)$.

It is very well known that this element severely locks, that is, the element is too stiff in bending-dominated shell problems. Of course, the strain field of this element is spatially isotropic.
- The MITC3 element.

With the assumption that the transverse shear strain be constant along the element edges, we construct the assumed transverse shear strain field for the MITC3 element as [9]

$\tilde{e}_{r \xi}=e_{r \xi}^{(1)}+c s, \quad \tilde{e}_{s \xi}=e_{s \xi}^{(2)}-c r \quad$ with

$c=e_{s \xi}^{(2)}-e_{r \xi}^{(1)}-e_{s \xi}^{(3)}+e_{r \xi}^{(3)}$

and use the tying points shown in Fig. 2. In Eq. (7), $e_{r \xi}^{(n)}$ and $e_{s \xi}^{(n)}$ are the covariant transverse shear strains of Eq. (6) at tying point $n$. Note that the assumed transverse shear strain field in Eq. (7) is spatially isotropic.

- The SRI3 element.

The covariant transverse shear strain field of the SRI3 shell element is assumed constant and given by Eq. (6)

$\hat{e}_{r \xi}=e_{r \xi}(1 / 3,1 / 3, \xi), \quad \hat{e}_{s \xi}=e_{s \xi}(1 / 3,1 / 3, \xi)$.

The transverse shear strain field of this element is of course also spatially isotropic. Note that this element stiffness matrix could be evaluated using one-point selective reduced integration for the transverse shear strains. However, this element displays a spurious zero energy mode and we would not use it in engineering analyses [1].

- The NIT3 element.

The three elements above are isotropic triangular shell finite elements. If we neglect the linear terms of the covariant transverse shear strains of the MITC3 element, a 3-node non-isotropic triangular shell finite element (referred herein as the NIT3 element) is obtained,

$\breve{e}_{r \xi}=e_{r \xi}^{(1)}, \quad \breve{e}_{s \xi}=e_{s \xi}^{(2)}$.

This is a natural attempt, because if we use two such elements to represent a rectangular domain similar tying positions can be selected as used for the MITC4 element (see Section 3). The third tying point is not required for the strain interpolations. This 3-node triangular shell finite element can also be derived using the "Discrete Shear Gap" concept and was referred to as the DSG3 element [12]. As for the SRI3 element, the NIT3 element also contains a spurious zero energy mode.

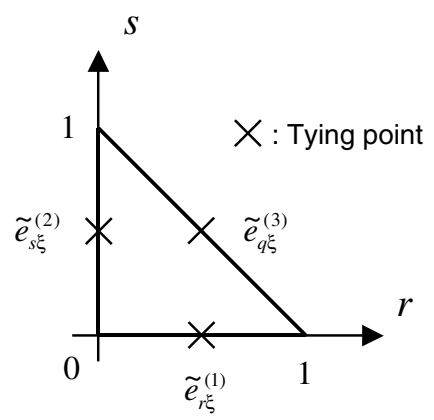

Fig. 2. Tying positions for the transverse shear strain of the MITC3 triangular shell element, $e_{q \xi}^{(3)}=\left(e_{s \xi}^{(3)}-e_{r \xi}^{(3)}\right) / \sqrt{2}$. 


\subsection{Transverse shear strain fields in flat geometry}

In this section, we study the transverse shear strain fields of the 3-node triangular shell finite elements assuming the elements are used in plate bending problems, merely to obtain insight into the element behaviors.

When the shell finite element is used for plate bending problems, with the plate of constant thickness defined in the $X Y$-plane, we have the conditions

$\vec{x}_{i}=\left\{\begin{array}{c}x_{i} \\ y_{i} \\ 0\end{array}\right\}, \quad \vec{u}_{i}=\left\{\begin{array}{c}0 \\ 0 \\ w_{i}\end{array}\right\}, \quad V_{n}^{i}=\vec{i}_{z}, \quad \vec{V}_{1}^{i}=\vec{i}_{x}, \quad \vec{V}_{2}^{i}=\vec{i}_{y}$

$$
\text { and } t_{i}=t \text { for all } i \text {, }
$$

where $\vec{i}_{x}, \vec{i}_{y}$ and $\vec{i}_{z}$ are the unit base vectors in the global Cartesian coordinate system.

From Eqs. (1) and (2), we then have the geometry and displacement interpolations

$$
\vec{x}=\left\{\begin{array}{c}
\sum_{i=1}^{3} h_{i} x_{i} \\
\sum_{i=1}^{3} h_{i} y_{i} \\
\frac{\xi}{2} t
\end{array}\right\}, \quad \vec{u}=\left\{\begin{array}{c}
\frac{\xi}{2} t \cdot \sum_{i=1}^{3} h_{i} \beta_{i} \\
-\frac{\xi}{2} t \cdot \sum_{i=1}^{3} h_{i} \alpha_{i} \\
\sum_{i=1}^{3} h_{i} w_{i}
\end{array}\right\} .
$$

We next consider the four different 3-node triangular shell elements mentioned in the previous section for plate bending problems.

- The QUAD3 element.

Using Eq. (11) in Eq. (6), we obtain the transverse shear strain field of the 3-node displacement-based shell finite element

$$
\begin{aligned}
& e_{r \xi}=\frac{t}{4}\left[w_{2}-w_{1}+\left(x_{2}-x_{1}\right) \cdot \sum_{i=1}^{3} h_{i} \beta_{i}-\left(y_{2}-y_{1}\right) \cdot \sum_{i=1}^{3} h_{i} \alpha_{i}\right], \\
& e_{s \xi}=\frac{t}{4}\left[w_{3}-w_{1}+\left(x_{3}-x_{1}\right) \cdot \sum_{i=1}^{3} h_{i} \beta_{i}-\left(y_{3}-y_{1}\right) \cdot \sum_{i=1}^{3} h_{i} \alpha_{i}\right] .
\end{aligned}
$$

\section{- The MITC3 element.}

Using Eq. (7), the transverse shear strain field of the MITC3 shell finite element is obtained

$$
\begin{aligned}
& \tilde{e}_{r \xi}=\frac{t}{4}\left[w_{2}-w_{1}+\frac{1}{2}\left(x_{2}-x_{1}\right)\left(\beta_{1}+\beta_{2}\right)-\frac{1}{2}\left(y_{2}-y_{1}\right)\left(\alpha_{1}+\alpha_{2}\right)\right]+c s, \\
& \tilde{e}_{s \xi}=\frac{t}{4}\left[w_{3}-w_{1}+\frac{1}{2}\left(x_{3}-x_{1}\right)\left(\beta_{1}+\beta_{3}\right)-\frac{1}{2}\left(y_{3}-y_{1}\right)\left(\alpha_{1}+\alpha_{3}\right)\right]-c r
\end{aligned}
$$

with

$$
\begin{aligned}
c= & \frac{t}{8}\left[\left(y_{2}-y_{3}\right) \alpha_{1}+\left(y_{3}-y_{1}\right) \alpha_{2}+\left(y_{1}-y_{2}\right) \alpha_{3}+\left(x_{3}-x_{2}\right) \beta_{1}\right. \\
& \left.+\left(x_{1}-x_{3}\right) \beta_{2}+\left(x_{2}-x_{1}\right) \beta_{3}\right] .
\end{aligned}
$$

- The SRI3 element.

Similarly, the SRI3 shell finite element has the transverse shear strains

$$
\begin{aligned}
\hat{e}_{r \xi}=\left.e_{r \xi}\right|_{r=s=1 / 3}= & \frac{t}{4}\left[w_{2}-w_{1}+\frac{1}{3}\left(x_{2}-x_{1}\right)\left(\beta_{1}+\beta_{2}+\beta_{3}\right)\right. \\
& \left.-\frac{1}{3}\left(y_{2}-y_{1}\right)\left(\alpha_{1}+\alpha_{2}+\alpha_{3}\right)\right], \\
\hat{e}_{s \xi}=\left.e_{s \xi}\right|_{r=s=1 / 3}= & \frac{t}{4}\left[w_{3}-w_{1}+\frac{1}{3}\left(x_{3}-x_{1}\right)\left(\beta_{1}+\beta_{2}+\beta_{3}\right)\right. \\
& \left.-\frac{1}{3}\left(y_{3}-y_{1}\right)\left(\alpha_{1}+\alpha_{2}+\alpha_{3}\right)\right] .
\end{aligned}
$$

- The NIT3 element.

Using Eq. (9), the transverse shear strain field of the NIT3 shell finite element is

$$
\begin{aligned}
\breve{e}_{r \xi}= & \frac{t}{4}\left[w_{2}-w_{1}+\frac{1}{2}\left(x_{2}-x_{1}\right)\left(\beta_{1}+\beta_{2}\right)\right. \\
& \left.-\frac{1}{2}\left(y_{2}-y_{1}\right)\left(\alpha_{1}+\alpha_{2}\right)\right], \\
\breve{e}_{s \xi}= & \frac{t}{4}\left[w_{3}-w_{1}+\frac{1}{2}\left(x_{3}-x_{1}\right)\left(\beta_{1}+\beta_{3}\right)\right. \\
& \left.-\frac{1}{2}\left(y_{3}-y_{1}\right)\left(\alpha_{1}+\alpha_{3}\right)\right] .
\end{aligned}
$$

\section{Convergence studies}

Using a reliable finite element discretization scheme, the finite element solution converges to the exact solution of the underlying mathematical model as the element size decreases $[2,3,13]$. However, it is important to use an appropriate norm to measure the convergence of the finite element solutions.

We use the s-norm proposed by Hiller and Bathe as a norm to measure convergence for mixed formulations [11]

||$\vec{u}-\left.\vec{u}_{h}\right|_{s} ^{2}=\int_{\Omega} \Delta \vec{\varepsilon}^{\mathrm{T}} \Delta \vec{\sigma} \mathrm{d} \Omega$,

where $\vec{u}$ denotes the exact solution and $\vec{u}_{h}$ denotes the solution of the finite element discretization. Here, $\vec{\varepsilon}$ and $\vec{\sigma}$ are the strain vector and the stress vector in the global Cartesian coordinate system, respectively, defined by

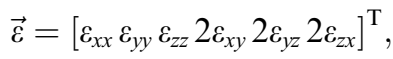

$\vec{\sigma}=\left[\sigma_{x x} \sigma_{y y} \sigma_{z z} \sigma_{x y} \sigma_{y z} \sigma_{z x}\right]^{\mathrm{T}}$

and

$\Delta \vec{\varepsilon}=\vec{\varepsilon}-\vec{\varepsilon}_{h}=\vec{\varepsilon}(\vec{x})-\mathbf{B}_{h}\left(\vec{x}_{h}\right) \mathbf{U}_{h}$,

$\Delta \vec{\sigma}=\vec{\sigma}-\vec{\sigma}_{h}=\vec{\sigma}(\vec{x})-\mathbf{C}_{h}\left(\vec{x}_{h}\right) \mathbf{B}_{h}\left(\vec{x}_{h}\right) \mathbf{U}_{h}$,

where $\mathbf{C}$ denotes the material stress-strain matrix, $\mathbf{B}$ is the strain-displacement operator and $\mathbf{U}$ is the vector of nodal degrees of freedom. The position vectors $\vec{x}$ and $\vec{x}_{h}$ 
correspond to the continuum domain and the discretized domain, respectively, and we have

$\vec{x}=\Pi\left(\vec{x}_{h}\right)$,

where $\Pi$ defines a one-to-one mapping.

In the practical use of this norm, a reliable finite element solution using a very fine mesh, $\vec{u}_{\text {ref }}$, can be employed instead of the exact solution. Using the reference solution, the s-norm in Eq. (17) is

$\left\|\vec{u}_{\mathrm{ref}}-\vec{u}_{h}\right\|_{s}^{2}=\int_{\Omega_{\mathrm{ref}}} \Delta \vec{\varepsilon}^{\mathrm{T}} \Delta \vec{\sigma} \mathrm{d} \Omega_{\mathrm{ref}}$,

with

$\Delta \vec{\varepsilon}=\vec{\varepsilon}_{\text {ref }}-\vec{\varepsilon}_{h}=\mathbf{B}_{\text {ref }}\left(\vec{x}_{\text {ref }}\right) \mathbf{U}_{\text {ref }}-\mathbf{B}_{h}\left(\vec{x}_{h}\right) \mathbf{U}_{h}$,

$\Delta \vec{\sigma}=\vec{\sigma}_{\text {ref }}-\vec{\sigma}_{h}=\mathbf{C}_{\text {ref }}\left(\vec{x}_{\text {ref }}\right) \mathbf{B}_{\text {ref }}\left(\vec{x}_{\text {ref }}\right) \mathbf{U}_{\text {ref }}-\mathbf{C}_{h}\left(\vec{x}_{h}\right) \mathbf{B}_{h}\left(\vec{x}_{h}\right) \mathbf{U}_{h}$

with $\vec{x}_{\text {ref }}=\Pi\left(\vec{x}_{h}\right)$.

To measure appropriately the performance of finite elements, it is necessary to study the relative error $E_{h}$ defined as
$E_{h}=\frac{\left\|\vec{u}_{\mathrm{ref}}-\vec{u}_{h}\right\|_{s}^{2}}{\left\|\vec{u}_{\mathrm{ref}}\right\|_{s}^{2}}$

For a uniformly-optimal (and hence non-locking) element we would have for any shell problem

$E_{h} \cong c h^{k}$,

where $c$ is independent of the shell thickness and $k=2$. As mentioned above such a 3-node element is very difficult to develop. But in such development, in order to properly see the qualities of an element, it is important to use an appropriate norm and to solve appropriate test problems decreasing the shell thickness $[9,11]$. A general numerical procedure to find the one-to-one mapping in Eq. (22) and to calculate the s-norm is proposed in Appendix.

Below we consider two problems for our convergence studies. We reported earlier that the MITC3 shell finite element shows excellent behavior in membrane-dominated problems [9]. Of course, the displacement-based 3-node shell finite element (QUAD3) gives optimal solutions in such problems. Since shell finite element solutions deteriorate in bending-dominated problems, we consider the fully
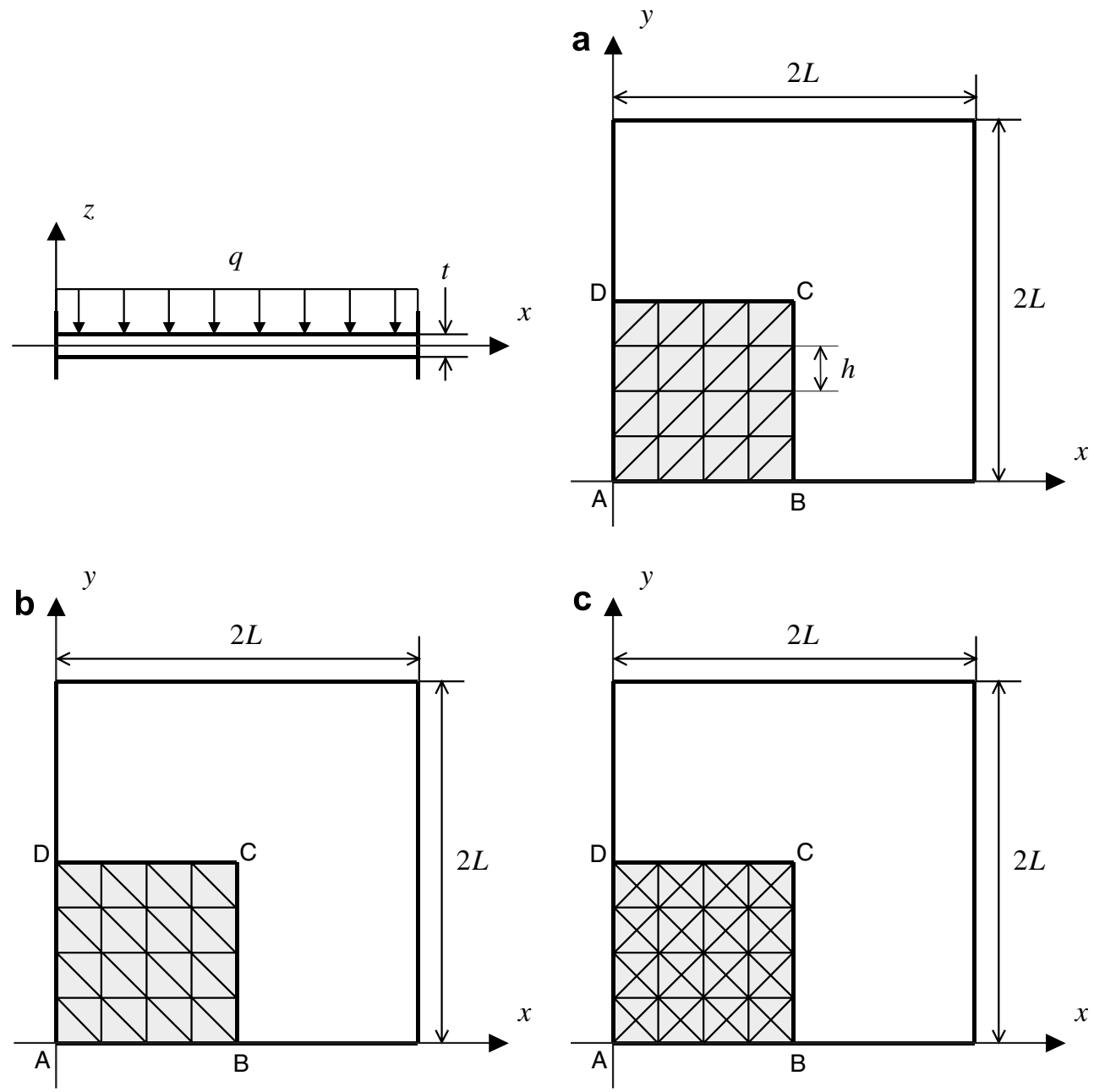

Fig. 3. Fully clamped plate under uniform pressure load ( $L=1.0, E=1.7472 \times 10^{7}, v=0.3$ and $\left.q=1.0\right)$. (a) and (b) Regular meshes (c) Cross mesh. 

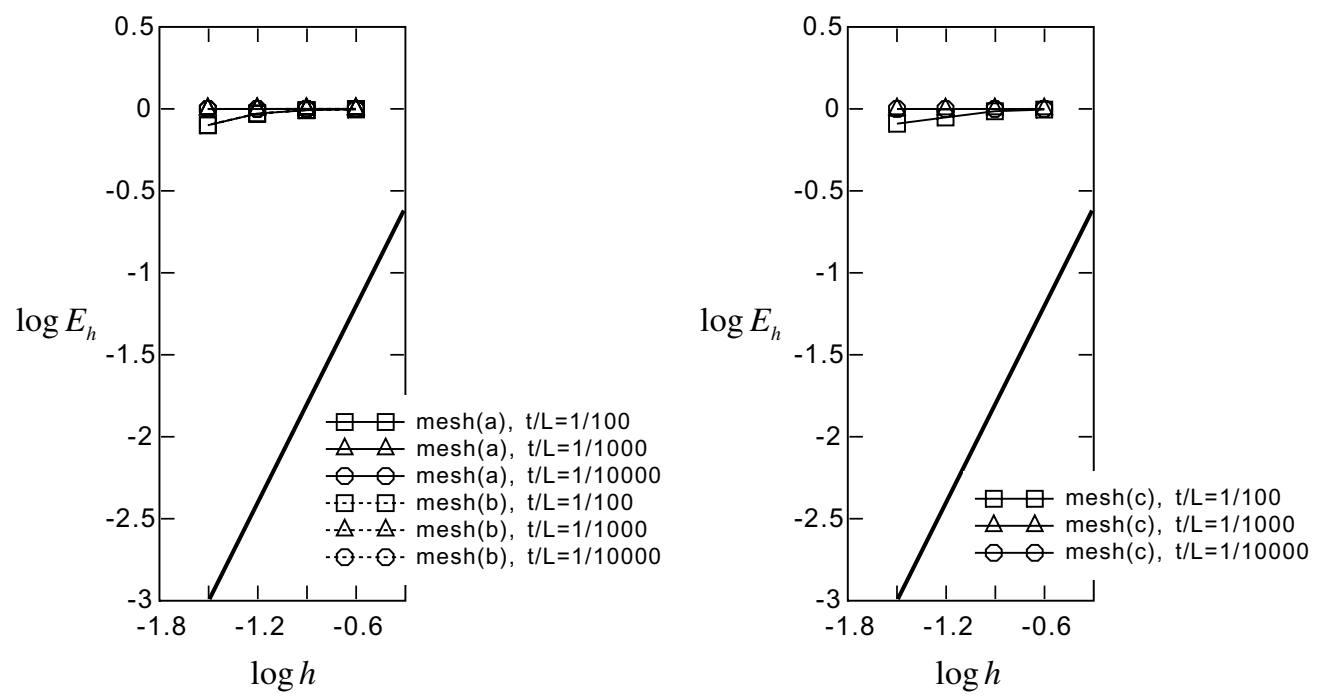

Fig. 4. Convergence curves of the QUAD3 shell finite element in the clamped plate problem (Left: regular mesh, Right: cross mesh).
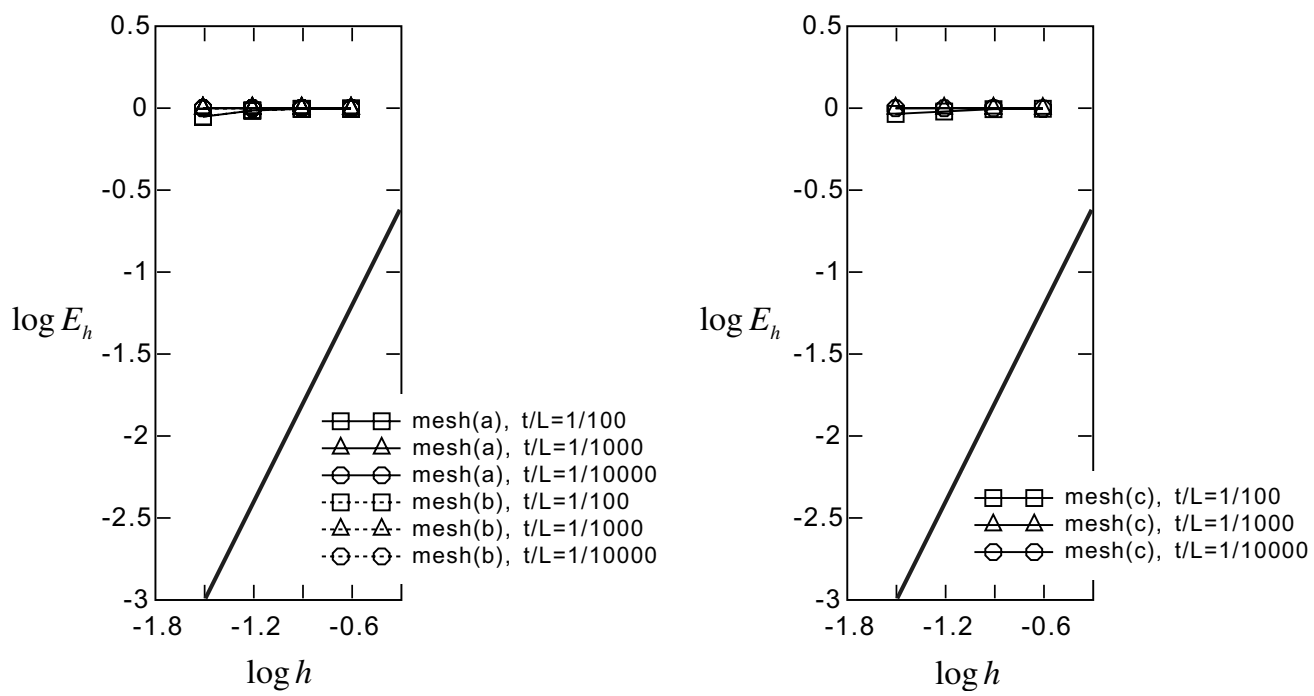

Fig. 5. Convergence curves of the SRI3 shell finite element in the clamped plate problem (Left: regular mesh, Right: cross mesh).

clamped plate problem and the hyperboloid shell problem (as already solved in less extensive convergence studies in Ref. [9]).

\subsection{Fully clamped plate problem}

We consider the plate bending problem shown in Fig. 3. The square plate of dimension $2 \mathrm{~L} \times 2 \mathrm{~L}$ with uniform thickness $t$ is subjected to a uniform pressure normal to the flat surface and all edges are fully clamped.

Due to symmetry, only one quarter model is considered (the region ABCD shown in Fig. 3) with the following symmetry and boundary conditions imposed: $u_{x}=\theta_{y}=0$ along BC, $u_{y}=\theta_{x}=0$ along DC and $u_{x}=u_{y}=u_{z}=\theta_{x}=\theta_{y}=0$ along $\mathrm{AB}$ and $\mathrm{AD}$.

Figs. 4-6 show the calculated convergence curves for the QUAD3, SRI3 and MITC3 shell finite elements, respec- tively, when $t / L=1 / 100,1 / 1000$ and $1 / 10,000$, for the three different mesh patterns of Figs. 3(a)-(c). Referring to the meshes in Fig. 3 as $4 \times 4$ element meshes, the convergence curves were obtained using $4 \times 4,8 \times 8,16 \times 16$ and $32 \times 32$ element meshes. The solutions were measured on the reference solutions, $\vec{u}_{\text {ref }}$ in Eq. (21), obtained using the MITC ${ }^{2}$ shell finite element with a mesh of $48 \times 48$ elements. The solid thick line represents the optimal convergence rate which can be obtained from 3-node triangular shell finite elements, $k=2$ in Eq. (24).

The QUAD3 and SRI3 shell finite elements severely lock in all the cases of thickness and mesh patterns considered. The MITC3 shell finite element locks in the meshes of Figs. 3(a) and (b) but the solution accuracy is still good

\footnotetext{
${ }^{2}$ The reliable performance of the MITC9 shell finite element is reported in Refs. $[2,11,14]$.
} 

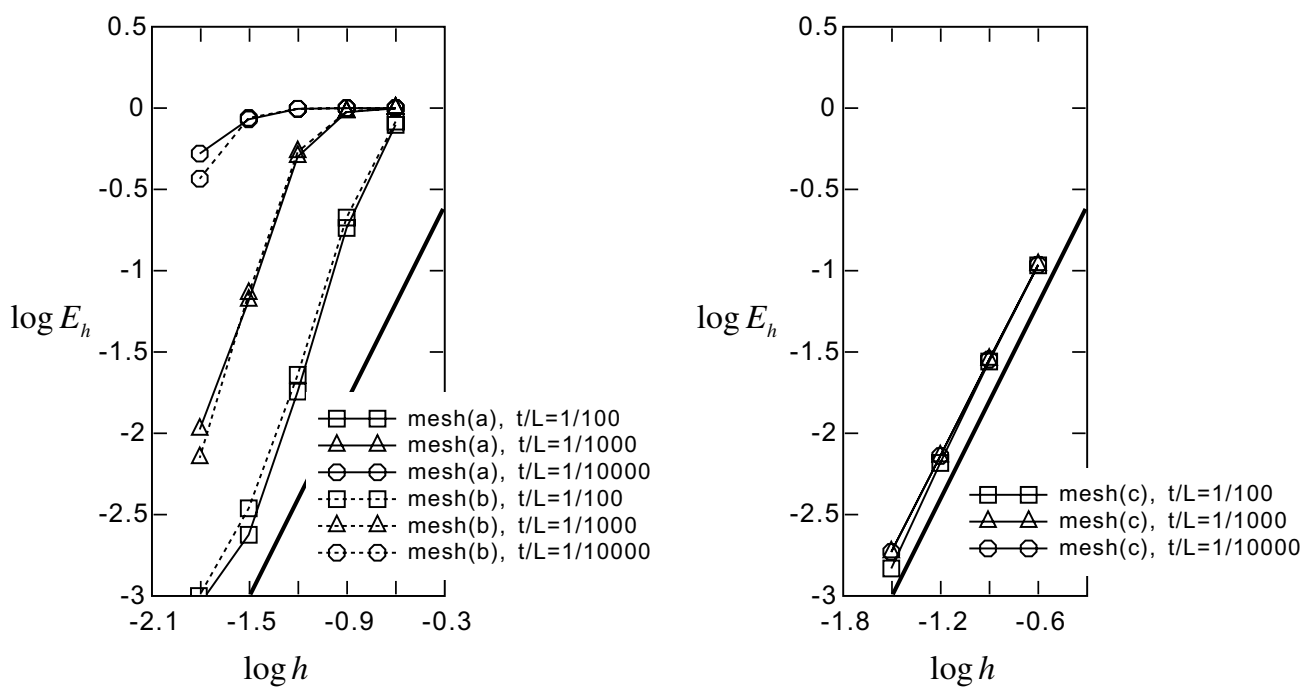

Fig. 6. Convergence curves of the MITC3 shell finite element in the clamped plate problem (Left: regular mesh, Right: cross mesh).

\section{X : Tying points of MITC4 element and element $\mathrm{A}$ \\ : Tying points of element $B$}
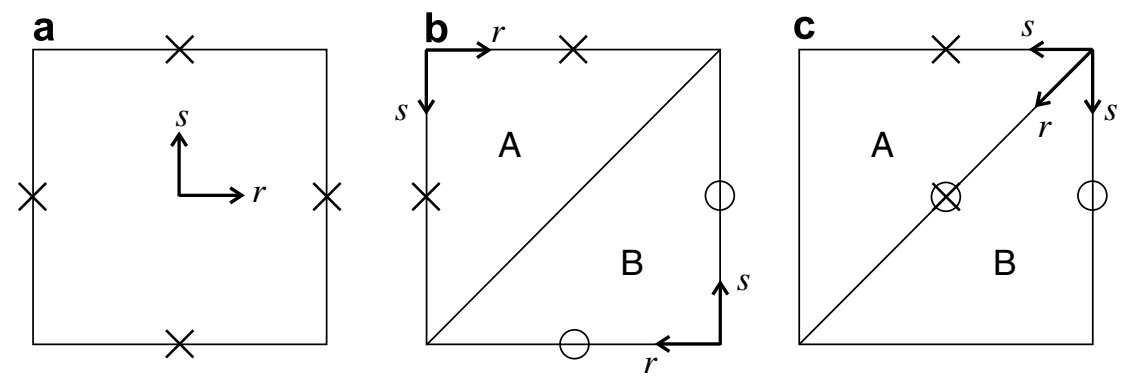

Fig. 7. Element orientations of the NIT3 element for the same mesh pattern: (a) Tying points of one MITC4 element, (b) Tying points of the NIT3 element in the element orientation-1, (c) Tying points of the NIT3 element in the element orientation-2.

up to $t / L=1 / 1000$. When the cross mesh in Fig. 3(c) is used, the MITC3 shell finite element shows the optimal convergence behavior independent of the shell thickness, and hence is uniformly optimal.

As mentioned above, it is interesting to investigate the convergence characteristics of the NIT3 shell finite element when different element orientations are used for the same mesh pattern. Since the element is not spatially isotropic, the behavior of the element depends on its orientation and the sequence of node numbering in a given mesh pattern. We use the mesh pattern of Fig. 3(a), see Fig. 7, and first select the element orientation in Fig. 7(b), which results in the tying points used in the MITC4 shell finite element, see Fig. 7(a). We then consider the element orientation in Fig. 7(c).

Fig. 8 displays the convergence curves of the NIT3 shell finite element when the element orientations of Figs. 7(b) and (c) are used. The element gives optimal convergence with the element orientation of Fig. 7(b) but locks with Fig. 7(c). Hence the NIT3 element shows different solution accuracy depending on the element orientation (with the same mesh pattern). Here, we do not show convergence results in other cases of mesh patterns and element orientations, but locking is observed for such cases as well. Of course this solution dependency on the element orientation in a given mesh pattern does not occur in isotropic shell finite elements.

\subsection{Hyperboloid shell problem}

This shell problem was also used in e.g. Refs. $[2,9,11,13]$ to study shell elements. The problem is described in Fig. 9. The midsurface of the shell structure is given by

$x^{2}+z^{2}=1+y^{2}, \quad y \in[-1,1]$

and the loading imposed is the smoothly varying periodic pressure normal to the surface

$p(\theta)=p_{0} \cos (2 \theta)$,

where $p_{0}=1.0$.

We consider the bending-dominated problem obtained when both ends are free. Using symmetry, the analyses 

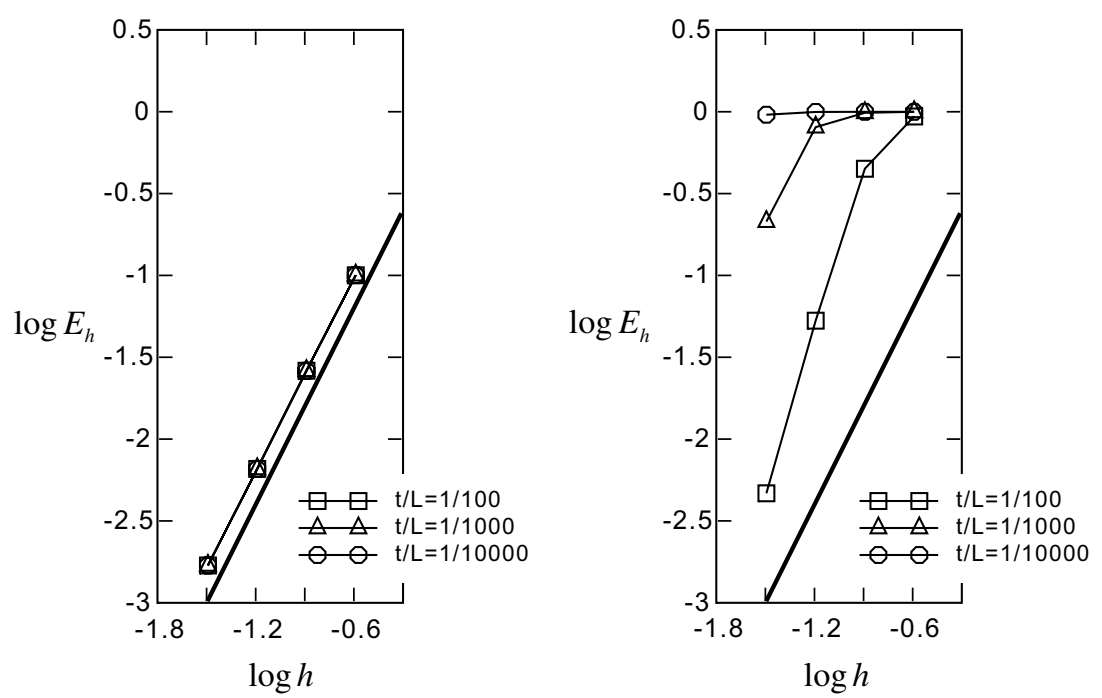

Fig. 8. Convergence curves of the NIT3 shell finite element in the clamped plate problem using the mesh pattern of Fig. 3(a): Left: element orientation-1 of Fig. 7(b), Right: element orientation-2 of Fig. 7(c).
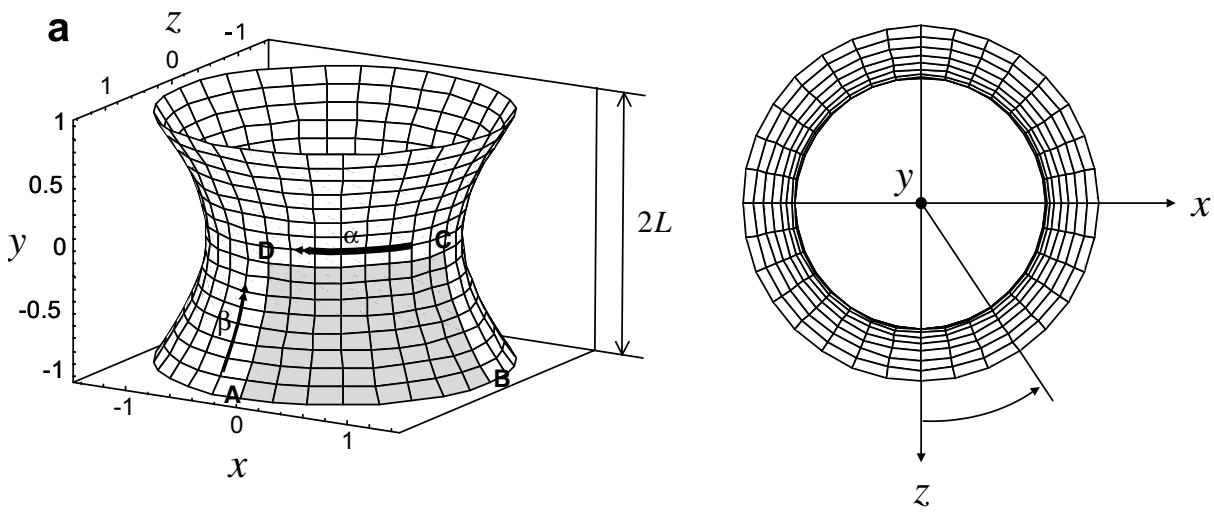

b
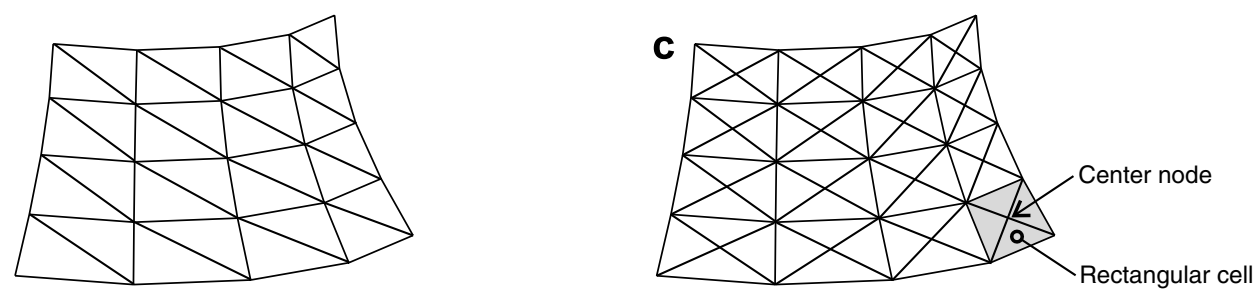

d
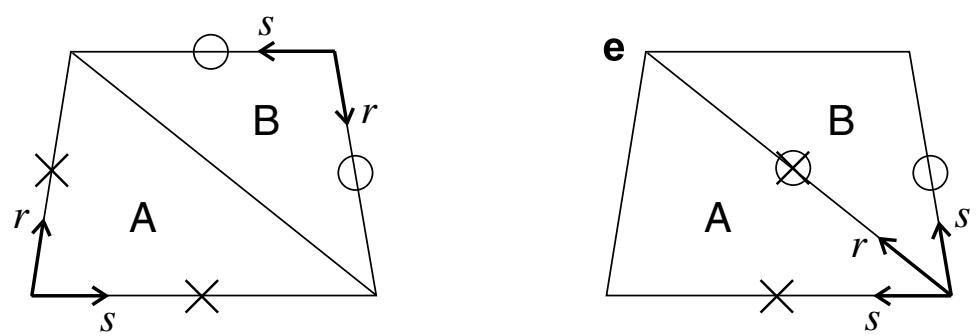

Fig. 9. Hyperboloid shell problem $\left(L=1.0, E=2.0 \times 10^{11}, v=1 / 3\right.$ and $\left.p_{0}=1.0\right)$ and mesh patterns used. (b) Regular mesh, (c) Cross mesh, (d) Tying points of the NIT3 element in the element orientation-1, (e) Tying points of the NIT3 element in the element orientation-2.

are performed using one eighth of the structure, the shaded region $\mathrm{ABCD}$ in Fig. 9(a). Considering the boundary conditions, we have: $u_{z}=\beta=0$ along $\mathrm{BC}, u_{x}=\beta=0$ along $\mathrm{AD}$ and $u_{y}=\alpha=0$ along DC.
For the convergence study we use the two different mesh patterns shown in Figs. 9(b) and (c) and the reference solution obtained with a mesh of $48 \times 48$ MITC9 shell finite elements. The very thin boundary layer is not specially 
meshed. For the mesh in Fig. 9(c), the center node positions of rectangular cells are evaluated from the average of the positions of the corners; hence the center nodes are not quite on the midsurface of the shell given in Eq. (25).

Figs. 10 and 11 show that the QUAD3 and SRI3 shell finite elements severely lock in the two types of meshes. Fig. 12 displays that, when the mesh of Fig. 9(b) is used, the MITC3 element also locks but the solution accuracy is useful in practice. When the mesh of Fig. 9(c) is used, the solutions using the MITC3 element become much better.

In Fig. 13, we report that the convergence of the NIT3 element is uniformly optimal with the element orientation-1 of Fig. 9(d) but a very different convergence behavior and much worse solution accuracy are obtained with the element orientation-2 of Fig. 9(e). It is important to note that there is a large difference in the solution accuracy depending on the element orientation in a given mesh pattern when this non-isotropic triangular shell finite element is used. We can expect a similar undesirable behavior for other non-isotropic triangular shell finite elements, a behavior that needs to be understood and taken into account, automatically or otherwise, in practical analyses.

\section{Simple bending test problems}

To this point, we presented the convergence behaviors of four different triangular shell finite elements depending on mesh patterns and element orientations used. Considering a cantilever plate problem and a two-sided clamped plate problem modeled with two or four elements, we next further study the transverse shear strain fields of the QUAD3, MITC3 and SRI3 triangular shell elements and the locking phenomenon in different meshes.
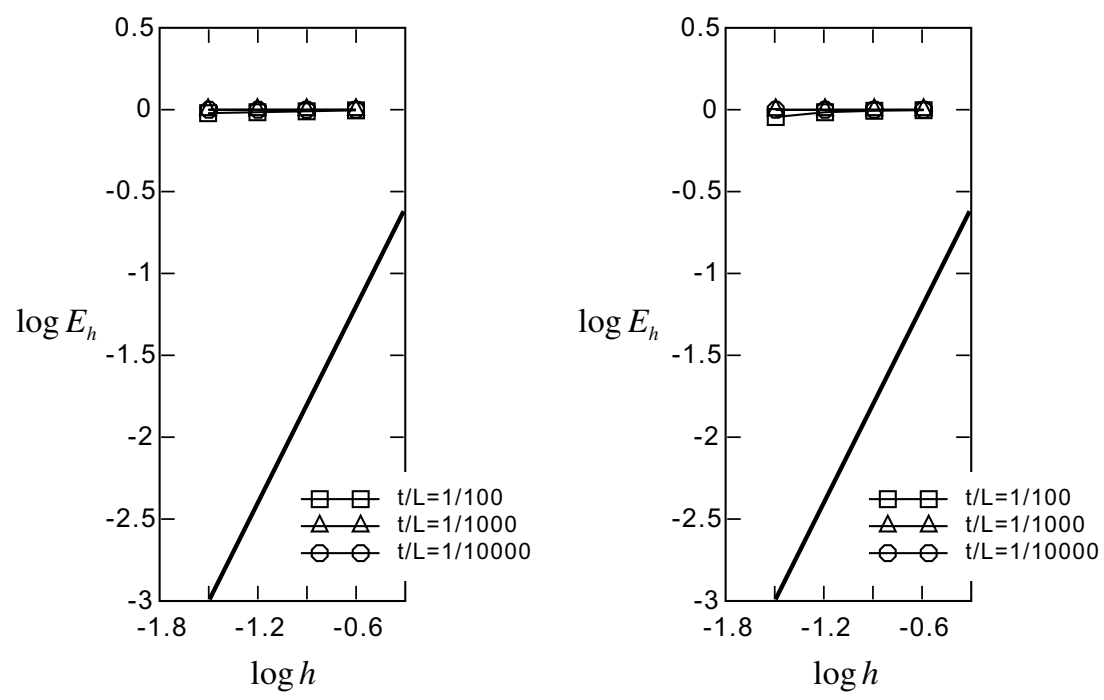

Fig. 10. Convergence curves of the QUAD3 shell finite element in the hyperboloid shell problem (Left: regular mesh, Right: cross mesh).
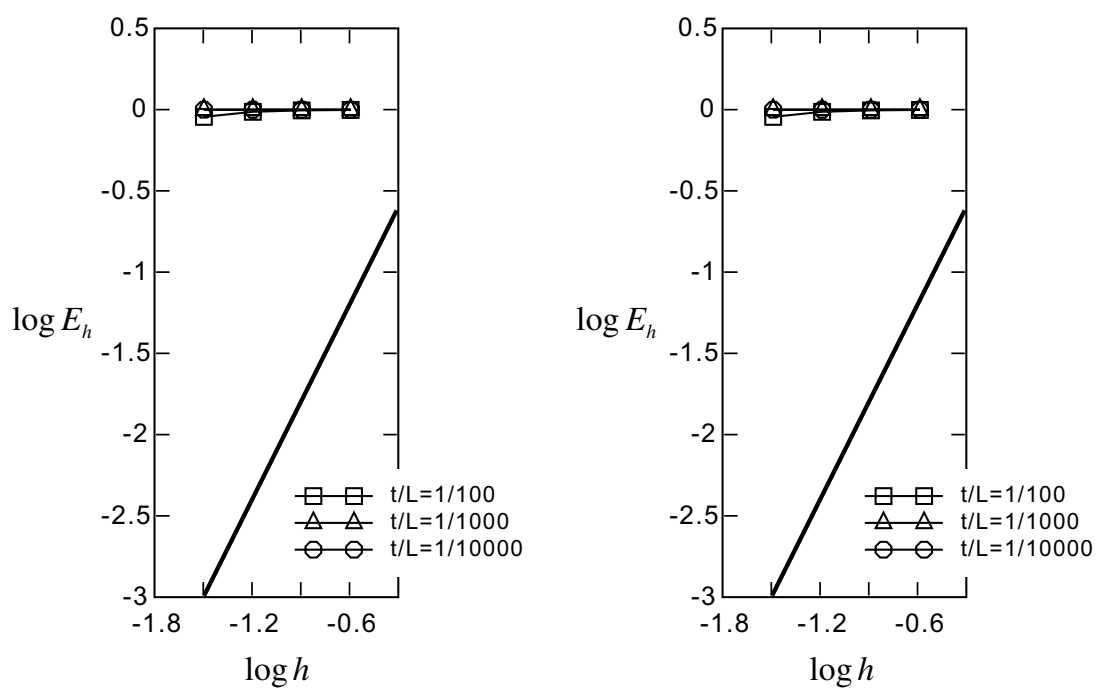

Fig. 11. Convergence curves of the SRI3 shell finite element in the hyperboloid shell problem (Left: regular mesh, Right: cross mesh). 

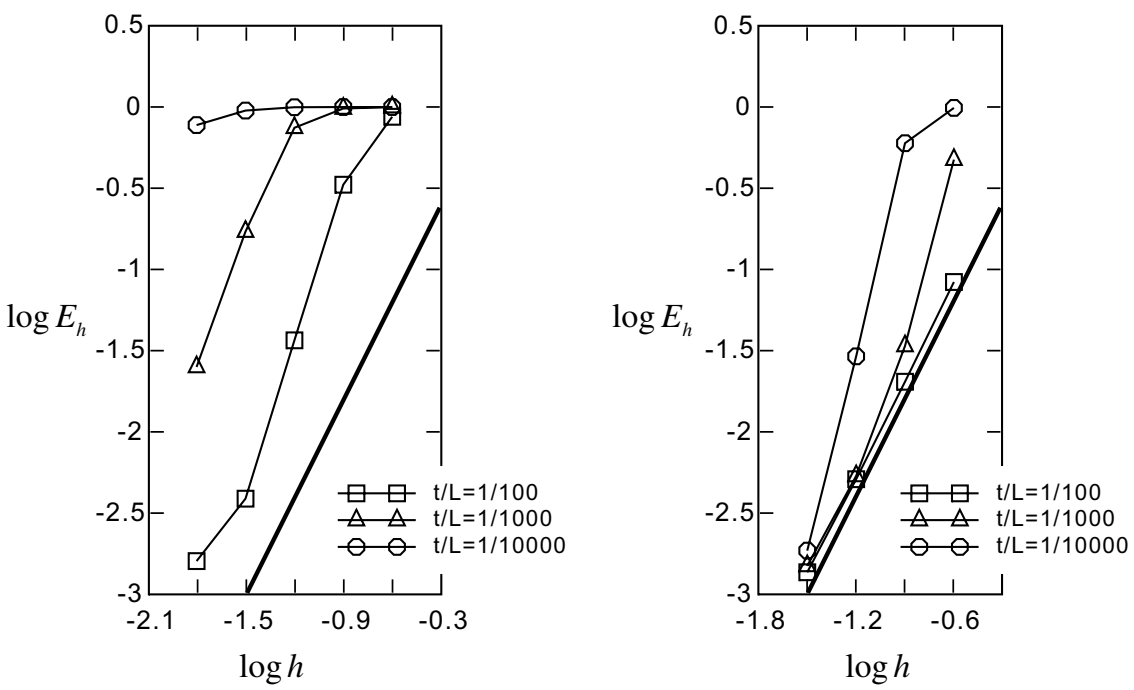

Fig. 12. Convergence curves of the MITC3 shell finite element in the hyperboloid shell problem (Left: regular mesh, Right: cross mesh).
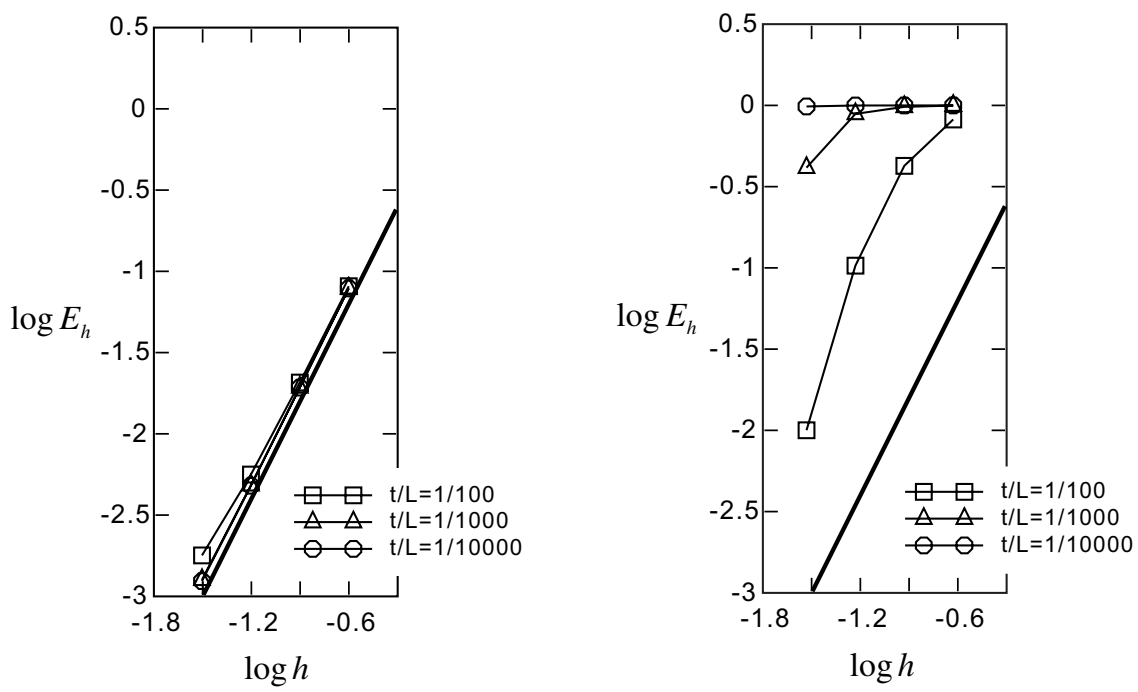

Fig. 13. Convergence curves of the NIT3 shell finite element in the hyperboloid shell problem using the mesh pattern of Fig. 9(b): Left: element orientation-1 of Fig. 9(d), Right: element orientation-2 of Fig. 9(e).

\subsection{Cantilever plate problem}

Let us consider the cantilever plate of dimension $L \times L$ shown in Fig. 14. The structure is subjected to a uniform moment $m_{\alpha}$ along its tip. This is a basic problem to test the pure bending behavior of beam/plate/shell finite elements.

We use only two triangular shell finite elements to solve the problem: element-I and element-II in Fig. 14 are used to examine in detail the behavior of the QUAD3, MITC3 and SRI3 shell finite elements.

The boundary condition of this plate problem along the clamped edge is

$w=\alpha=\beta=0$

and from the geometry, $x_{1}=1, y_{1}=0, \quad x_{2}=1, y_{2}=1$

and

$x_{3}=0, y_{3}=0$ for element-I,

$x_{1}=0, y_{1}=1, \quad x_{2}=1, y_{2}=1$

and

$x_{3}=0, y_{3}=0$ for element-II,

in which the subscripts are the element node numbers on the element level, see Figs. 1 and 14. Note that the node numbers used in the following equations correspond to the global node numbers given in Fig. 14.

This is a pure bending problem, that is, the exact analytical solution corresponds to

$e_{r \xi}=e_{s \xi}=0 \quad$ in $\Omega$, 

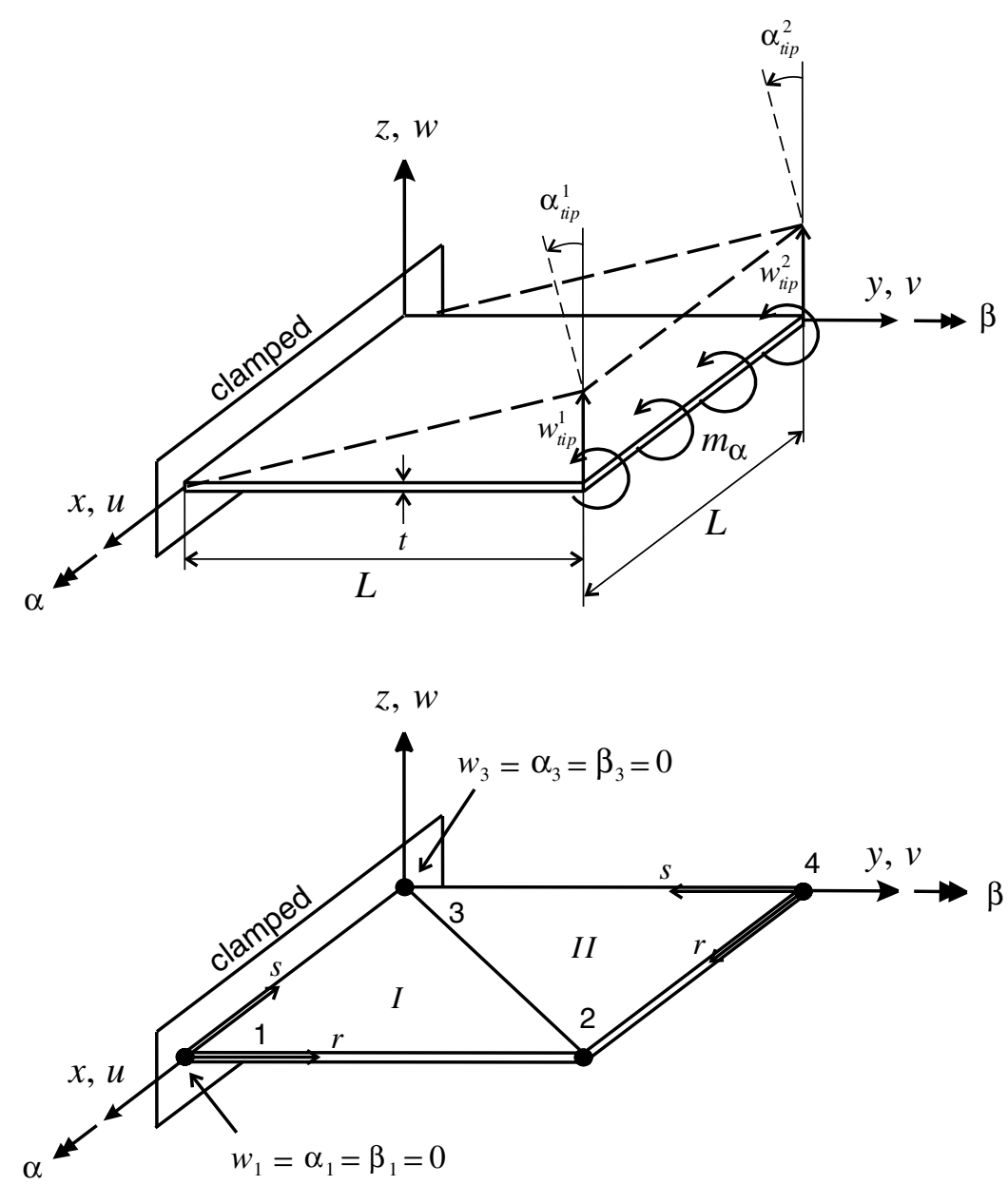

Fig. 14. Cantilever plate problem $\left(L=1.0, m_{\alpha}=2 / L, E=1.7472 \times 10^{7}, v=0.0\right)$. The rotations $\beta_{\text {tip }}^{1}$ and $\beta_{\text {tip }}^{2}$ should be zero and are for clarity not shown in the figure.

where $\Omega$ is the whole plate domain including the elements-I and -II, and the theoretical relationship between the deflection $w_{\text {tip }}$, rotation $\alpha_{\text {tip }}$ and rotation $\beta_{\text {tip }}$, which can be calculated from basic mechanics, is given by

$w_{\text {tip }}=\frac{1}{2} \alpha_{\text {tip }}, \quad \beta_{\text {tip }}=0$.

There is no anticlastic curvature since Poisson's ratio $v=0.0$. Hence the exact solutions for the nodal rotations and displacements in Fig. 14 must correspond to Eq. (30). We now summarize the resulting transverse shear strain fields obtained in the solution of this problem for the three shell finite elements.

- The QUAD3 element.

Using the conditions in Eqs. (27) and (28) with Eq. (12), we obtain the transverse shear strain field of element-I,

$e_{r \xi}^{\mathrm{I}}=\frac{t}{4}\left(w_{2}-\alpha_{2} r\right), \quad e_{s \xi}^{\mathrm{I}}=-\frac{t}{4} \beta_{2} r$,

and the transverse shear strain field of element-II, $e_{r \xi}^{\mathrm{II}}=\frac{t}{4}\left(w_{2}-w_{4}+h_{1} \beta_{4}+h_{2} \beta_{2}\right)$,

$e_{s \xi}^{\mathrm{II}}=\frac{t}{4}\left(-w_{4}+h_{1} \alpha_{4}+h_{2} \alpha_{2}\right)$.

Note that, in Eqs. (31) and (32), $r$ and $s$ are independently defined in each element, that is, $r$ and $s$ in element-I are independent of $r$ and $s$ in element-II.

A necessary condition, but not sufficient, for an element to avoid locking is that the transverse shear strain field can express the pure bending condition in Eq. (29) with the solution in Eq. (30). For the QUAD3 element, however, the solution in Eq. (30) does not make the transverse shear strain field in Eqs. (31) and (32) vanish in elements-I and -II and therefore locking is expected in this case.

- The MITC3 element.

Similarly, we obtain for the MITC3 element

$$
\begin{aligned}
\tilde{e}_{r \xi}^{\mathrm{I}} & =\frac{t}{4}\left(w_{2}-\frac{1}{2} \alpha_{2}\right)+c^{\mathrm{I}} s, \quad \tilde{e}_{s \xi}^{\mathrm{I}}=-c^{\mathrm{I}} r \quad \text { with } \\
c^{\mathrm{I}} & =\frac{t}{8} \beta_{2},
\end{aligned}
$$

and 
$\tilde{e}_{r \xi}^{\mathrm{II}}=\frac{t}{4}\left(w_{2}-w_{4}+\frac{1}{2} \beta_{4}+\frac{1}{2} \beta_{2}\right)+c^{\mathrm{II}} s$,

$\tilde{e}_{s \xi}^{\mathrm{II}}=\frac{t}{4}\left(-w_{4}+\frac{1}{2} \alpha_{4}\right)-c^{\mathrm{II}} r \quad$ with

$c^{\mathrm{II}}=\frac{t}{8}\left(\alpha_{4}-\alpha_{2}-\beta_{4}\right)$

Using the condition in Eq. (29), we obtain

$$
\begin{aligned}
& w_{2}-\frac{1}{2} \alpha_{2}=0, \quad \beta_{2}=0, \quad w_{2}-w_{4}+\frac{1}{2} \beta_{4}+\frac{1}{2} \beta_{2}=0, \\
& -w_{4}+\frac{1}{2} \alpha_{4}=0, \quad \alpha_{4}-\alpha_{2}-\beta_{4}=0,
\end{aligned}
$$

and Eq. (30) satisfies Eq. (35), that is,

$w_{4}=w_{2}=\frac{1}{2} \alpha_{4}=\frac{1}{2} \alpha_{2}, \quad \beta_{4}=\beta_{2}=0$.

Therefore, Eq. (30) is a solution of Eq. (35). As a result, the MITC3 shell finite element can express the pure bending condition.

- The SRI3 element.

For the SRI3 element we obtain

$\hat{e}_{r \xi}^{\mathrm{I}}=\frac{t}{4}\left(w_{2}-\frac{1}{3} \alpha_{2}\right), \quad \hat{e}_{s \xi}^{\mathrm{I}}=-\frac{t}{12} \beta_{2}$

and

$\hat{e}_{r \xi}^{\mathrm{II}}=\frac{t}{4}\left(w_{2}-w_{4}+\frac{1}{3} \beta_{4}+\frac{1}{3} \beta_{2}\right)$,

$\hat{e}_{s \xi}^{\mathrm{II}}=\frac{t}{4}\left(-w_{4}+\frac{1}{3} \alpha_{4}+\frac{1}{3} \alpha_{2}\right)$,

and the analysis shows that the SRI3 shell finite element can also not predict the pure bending displacement in Eq. (30) and, indeed, the transverse shear strains vanish with

$$
\begin{aligned}
& w_{2}-\frac{1}{3} \alpha_{2}=0, \quad \beta_{2}=0, \quad \alpha_{4}-\beta_{4}=0, \\
& w_{4}-\frac{1}{3} \beta_{4}+\frac{1}{3} \alpha_{2}=0
\end{aligned}
$$

leading to locking.

We next calculate the numerical results using the three shell finite elements for the cantilever plate problem with $L=1, m_{\alpha}=2 / L, E=1.7472 \times 10^{7}$ and $v=0.0$. Table 1 shows the tip deflections and rotations when $t / L=1 / 1000$.

As expected, Table 1 shows that the MITC3 shell finite element produces the theoretical tip displacements and

Table 1

Tip displacements of the cantilever plate problem $(t / L=1 / 1000)$

\begin{tabular}{lllll}
\hline & QUAD3 & MITC3 & SRI3 & Theoretical value \\
\hline$w_{\text {tip }}^{1}$ & $1.27650 \mathrm{E}-03$ & $6.86813 \mathrm{E}+02$ & $4.57876 \mathrm{E}+02$ & $6.86813 \mathrm{E}+02$ \\
$w_{\text {tip }}^{2}$ & $2.24774 \mathrm{E}-03$ & $6.86813 \mathrm{E}+02$ & $6.86814 \mathrm{E}+02$ & $6.86813 \mathrm{E}+02$ \\
$\alpha_{\text {tip }}^{1}$ & $3.13575 \mathrm{E}-03$ & $1.37363 \mathrm{E}+03$ & $1.37363 \mathrm{E}+03$ & $1.37363 \mathrm{E}+03$ \\
$\alpha_{\text {tip }}^{2}$ & $4.30124 \mathrm{E}-03$ & $1.37363 \mathrm{E}+03$ & $6.86816 \mathrm{E}+02$ & $1.37363 \mathrm{E}+03$ \\
$\beta_{\text {tip }}^{1}$ & $5.54999 \mathrm{E}-04$ & $0.00000 \mathrm{E}+00$ & $2.06043 \mathrm{E}-03$ & $0.00000 \mathrm{E}+00$ \\
$\beta_{\text {tip }}^{2}$ & $1.66499 \mathrm{E}-03$ & $0.00000 \mathrm{E}+00$ & $6.86811 \mathrm{E}+02$ & $0.00000 \mathrm{E}+00$ \\
\hline
\end{tabular}

Table 2

Strain energies for the cantilever plate problem

\begin{tabular}{llll}
\hline$t / L$ & QUAD3 & MITC3 & SRI3 \\
\hline $1 / 100$ & $3.71728 \mathrm{E}-04$ & $1.37363 \mathrm{E}+00$ & $1.03040 \mathrm{E}+00$ \\
$1 / 1000$ & $3.71849 \mathrm{E}-03$ & $1.37363 \mathrm{E}+03$ & $1.03022 \mathrm{E}+03$ \\
\hline
\end{tabular}

rotations, whereas the QUAD3 and SRI3 elements do not give good results. Table 1 also shows that the QUAD3 and SRI3 elements each produce different displacements at both tips and the nodal variables of the SRI3 element satisfy the above conditions. Note that excellent results would be obtained for any ratio of $t / L$ using the MITC3 element. Also, if the cross mesh pattern of the MITC3 shell element is used for this cantilever plate problem, the same excellent results are obtained.

The cantilever plate problem is a bending-dominated problem and the strain energy $U$ stored in the structure is a function of the thickness, see Ref. [4],

$U(t) \propto t^{-3}$.

Table 2 shows the strain energies of the cantilever plate problem for the thickness parameters $t / L=1 / 100$ and $t / L=1 / 1000$. As expected, the numerical results show that the MITC3 shell finite element works well in this plate problem but the QUAD3 element locks. Note that, although the SRI3 element is based on a transverse shear strain interpolation of lower order than the MITC3 element, the MITC3 element gives a more flexible bending behavior.

\subsection{Two-sided clamped plate problem}

We observed that in the cantilever plate problem the MITC3 shell finite element does not lock, but it shows some locking in the fully clamped plate problem and the hyperboloid shell problem. Considering a simple two-sided clamped plate problem, we further investigate the behavior of the MITC3 shell finite element.

The clamped plate of dimension $L \times L$ shown in Fig. 15 is subjected to uniform moments $m_{\alpha}$ and $-m_{\beta}$ along its free sides. The boundary conditions are $w=\alpha=\beta=0$ along the clamped edges. We consider three different meshes, mesh A, mesh B and mesh $\mathrm{C}$ as shown in Fig. 15.

Table 3 presents the strain energies calculated using the MITC3 shell finite element. The results show that the MITC3 element works well in the meshes B and C, but locks when using the mesh A.

Considering the mesh $\mathrm{A}$ and using the notation of Section 4.1, the boundary conditions are

$\alpha_{1}=\alpha_{3}=\alpha_{4}=\beta_{1}=\beta_{3}=\beta_{4}=w_{1}=w_{3}=w_{4}=0$.

The transverse shear strain field is given by

$\tilde{e}_{r \xi}^{\mathrm{I}}=\frac{t}{4}\left(w_{2}-\frac{1}{2} \alpha_{2}\right)+c^{\mathrm{I}} s, \quad \tilde{e}_{s \xi}^{\mathrm{I}}=-c^{\mathrm{I}} r \quad$ with $c^{\mathrm{I}}=\frac{t}{8} \beta_{2}$ 


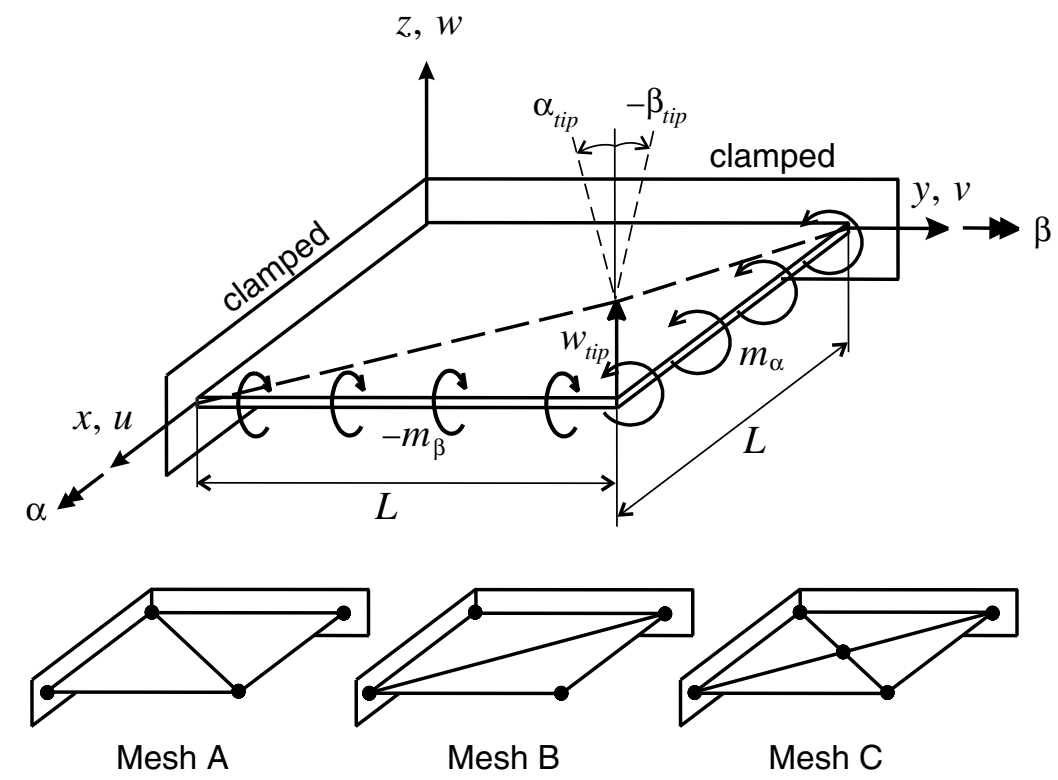

Fig. 15. Two-sided clamped plate $\left(L=1.0, m_{\alpha}=m_{\beta}=2 / L, E=1.7472 \times 10^{7}, v=0.0\right)$.

Table 3

Strain energies using the MITC3 element for the two-sided clamped plate problem

\begin{tabular}{llll}
\hline$t / L$ & Mesh A & Mesh B & Mesh C \\
\hline $1 / 100$ & $4.11903 \mathrm{E}-04$ & $6.86813 \mathrm{E}-01$ & $6.86937 \mathrm{E}-01$ \\
$1 / 1000$ & $4.12086 \mathrm{E}-03$ & $6.86813 \mathrm{E}+02$ & $6.86814 \mathrm{E}+02$ \\
\hline
\end{tabular}

and

$\tilde{e}_{r \xi}^{\mathrm{II}}=\frac{t}{4}\left(w_{2}+\frac{1}{2} \beta_{2}\right)+c^{\mathrm{II}} s, \quad \tilde{e}_{s \xi}^{\mathrm{II}}=-c^{\mathrm{II}} r \quad$ with $c^{\mathrm{II}}=-\frac{t}{8} \alpha_{2}$.

Clearly, the only nodal displacements and rotations that satisfy the pure bending condition are

$\alpha_{2}=\beta_{2}=w_{2}=0$,

and this implies locking in this case.

Similarly, it is simple to show that the QUAD3 and SRI3 elements lock in this plate problem for all three meshes used.

These results are in correspondence with the finding that, while of course the QUAD3, SRI3, and MITC3 elements pass the membrane patch tests, of these elements only the MITC3 element passes also the bending patch test [1].

\section{Conclusions}

Our objective was to obtain insight into the convergence behavior of 3-node triangular shell finite elements in bending-dominated problems depending on the mesh patterns used. We reviewed the formulations of four 3-node triangular shell finite elements and their strain fields, the QUAD3, SRI3, MITC3 and NIT3 elements, and presented the solutions of a fully clamped plate problem and a hyperboloid shell problem. Although the SRI3 and NIT3 elements contain a spurious zero energy mode - and can therefore not be recommended for practical use - we evaluated these elements in our study merely to obtain insight into element behaviors.

Regarding the convergence behavior of the 3-node triangular shell finite elements, we have made the following observations:

- In the fully clamped plate problem, the QUAD3 and SRI3 shell finite elements severely lock regardless of the mesh patterns used. In the regular meshes, the MITC3 element shows some locking but frequently acceptable for practical analysis, and when the cross mesh of the MITC3 shell finite element is used, almost optimal convergence is obtained. The NIT3 shell finite element shows optimal convergence when for a specific mesh pattern the tying points of the element are aligned as used in the MITC4 element, but locking is seen in other cases, that is, the solution accuracy given by the NIT3 element highly depends on the mesh patterns used and on the element orientation in a given mesh pattern.

- In the hyperboloid shell problem, severe locking is seen for the QUAD3 and SRI3 shell finite elements. The MITC3 shell finite element shows alleviated locking in the regular mesh and much better results in the cross mesh. For the NIT3 element, the results highly depend on the element orientation in a given mesh pattern.

We also studied the transverse shear strain fields and strain energies stored in two simple plate bending problems. This simple study gave some insight why there is the mesh-dependent behavior of the MITC3 shell finite element in the fully clamped plate problem and the hyperboloid shell problem. 
Based on the results of this study, it is obvious that for the benchmark tests of triangular shell finite elements, various mesh patterns need to be considered, and, in addition, when non-isotropic shell finite elements are tested, their performance should also be studied considering their various orientations in the given mesh patterns.

\section{Appendix. A general scheme for the numerical calculation of the s-norm}

We here propose a numerical procedure to calculate the s-norm for shell finite element solutions with general types of elements and general meshes.

The reference numerical solution given by $\vec{u}_{\text {ref }}$ is employed instead of the exact solution and the target numerical solution given by $\vec{u}_{h}$ is compared with this reference solution. The major difficulty of the s-norm calculation is to establish the mapping points between the reference mesh and the target mesh.

We evaluate the integration in Eq. (21) using the Gauss integration technique in the reference domain. Figs. 16(a) and (b) shows the reference mesh and the target mesh. In the figures, $\left(r_{\text {ref }}, s_{\text {ref }}, \xi_{\text {ref }}\right)$ are the isoparametric coordinates of the Gauss integration point of the element in the refer- ence mesh, and $\left(r_{h}, s_{h}, \xi_{h}\right)$ are the corresponding isoparametric coordinates of the corresponding element in the target mesh. Our goal is to find the corresponding element and to establish $\left(r_{h}, s_{h}\right)$ for the given $\left(r_{\text {ref }}, s_{\text {ref }}\right)$ because $\xi_{h}=\xi_{\text {ref }}$ in shells

The first step is to find the corresponding element in the target mesh. From the given $\left(r_{\text {ref }}, s_{\text {ref }}\right)$ of the element in the reference mesh, we calculate the global coordinates $\left(x_{\text {ref }}, y_{\text {ref }}, z_{\text {ref }}\right)$

$$
\begin{aligned}
& x_{\text {ref }}=\sum_{i=1}^{n_{\text {ref }}} h_{i}\left(r_{\text {ref }}, s_{\text {ref }}\right) x_{\text {ref }}^{i}, \quad y_{\text {ref }}=\sum_{i=1}^{n_{\text {ref }}} h_{i}\left(r_{\text {ref }}, s_{\text {ref }}\right) y_{\text {ref }}^{i}, \\
& z_{\text {ref }}=\sum_{i=1}^{n_{\text {ref }}} h_{i}\left(r_{\text {ref }}, s_{\text {ref }}\right) z_{\text {ref }}^{i},
\end{aligned}
$$

where $n_{\text {ref }}$ is the number of nodes of the element in the reference mesh. Then in the target mesh we find the element corresponding to the minimum of $\eta$

$\eta=\frac{\left|S-\sum_{i=1}^{m} S_{i}\right|}{S}$

Here $S$ is the area of the element tested in the target mesh, $S_{i}$ is the area of the triangle which consists of the point ( $\left.x_{\mathrm{ref}}, y_{\mathrm{ref}}, z_{\mathrm{ref}}\right)$ and the edge $i$ of that element in the target mesh and $m$ is the number of edges on the boundary of that
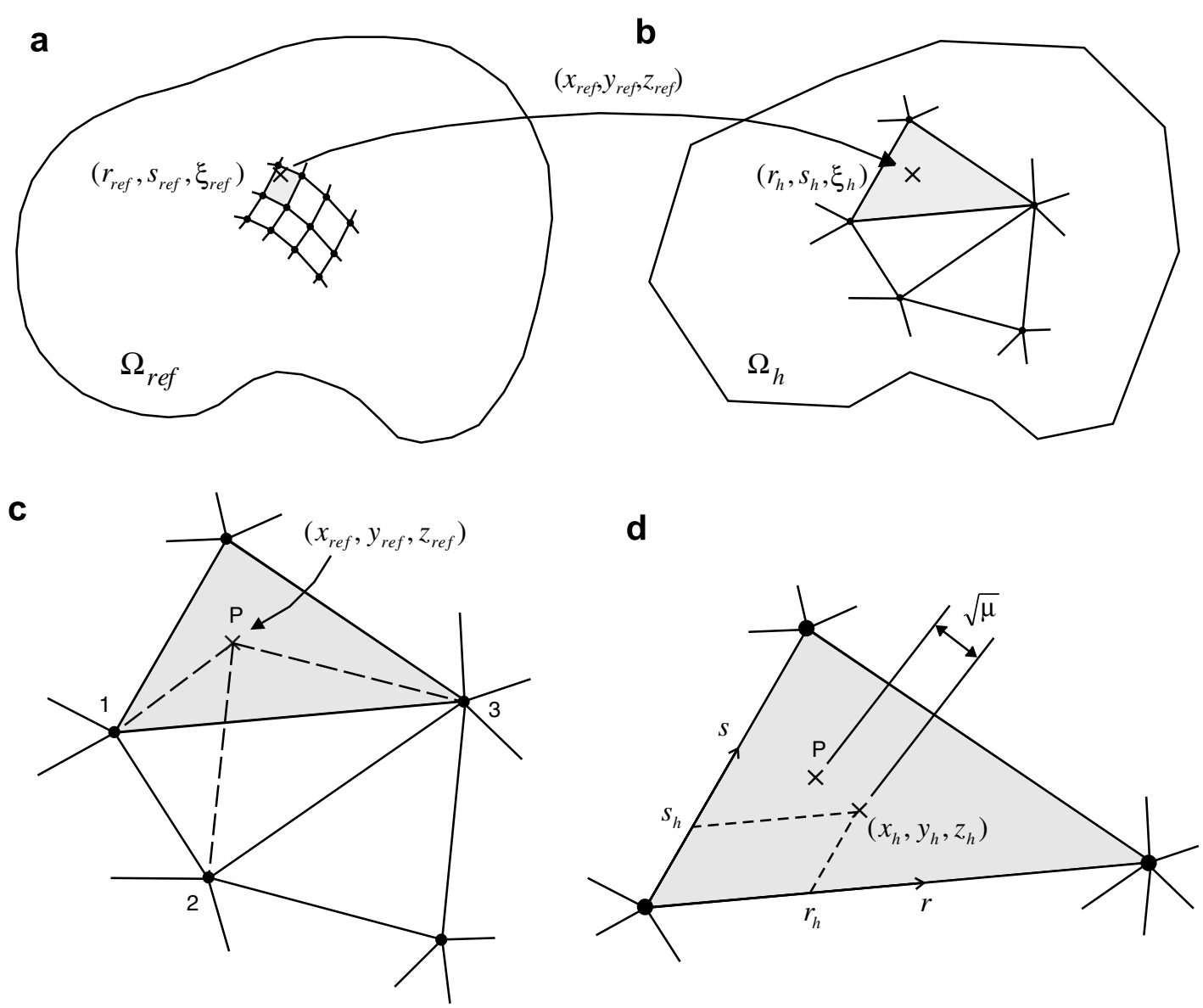

Fig. 16. Mapping between the reference mesh and the target mesh (The corresponding element in the target mesh is shaded in (b), (c) and (d).); (a) Reference mesh, (b) Target mesh, (c) Triangular areas of a tested 3-node element in the target mesh, (d) Finding $\left(r_{h}, s_{h}\right)$ in the corresponding element. 
element. For example, in Fig. 16, $S$ is given by the points 1-2-3 in Fig. 16(c), and $S_{1}$ (by points 1-2-P), $S_{2}$ (by points 2-3-P) and $S_{3}$ (by points 3-1-P) in Fig. 16(c) are the triangular areas for the tested 3-node element in the target mesh.

For a flat element, if the point ( $\left.x_{\text {ref }}, y_{\text {ref }}, z_{\text {ref }}\right)$ is inside the element, $\eta=0$; otherwise $\eta>0$. Considering general geometries, it is sufficient to find the element for which $\eta$ in Eq. (A.2) is minimized. To search for the element accurately in a general $n$-node element mesh, we divide each $n$-node element into triangular domains and proceed as above.

The second step is to find $\left(r_{h}, s_{h}\right)$ in the element that was just found, see Fig. 16(d). We need to establish the solution $\left(r_{h}, s_{h}\right)$ that minimizes

$\mu=\left(x_{h}-x_{\text {ref }}\right)^{2}+\left(y_{h}-y_{\text {ref }}\right)^{2}+\left(z_{h}-z_{\text {ref }}\right)^{2}$,

with

$x_{h}=\sum_{i=1}^{n_{h}} h_{i}\left(r_{h}, s_{h}\right) x_{h}^{i}, \quad y_{h}=\sum_{i=1}^{n_{h}} h_{i}\left(r_{h}, s_{h}\right) y_{h}^{i}$,

$z_{h}=\sum_{i=1}^{n_{h}} h_{i}\left(r_{h}, s_{h}\right) z_{h}^{i}$

where $n_{h}$ is the number of nodes of the corresponding element in the target mesh. We achieve this by employing, for example, a simple bisection algorithm [15].

Finally, we calculate the strains and stresses for $\left(r_{\text {ref }}\right.$, $\left.s_{\text {ref }}, \xi_{\text {ref }}\right)$ in the reference solution and for $\left(r_{h}, s_{h}, \xi_{h}\right)$ in the target solution and, by Eq. (21), the s-norm is obtained. This scheme can be used for shell finite element solutions (reference and target) with general types of elements and general meshes.

Also, of course

$\frac{1}{2}\left\|\vec{u}_{\text {ref }}\right\|_{s}^{2}=\frac{1}{2} \int_{\Omega_{\text {ref }}} \vec{\varepsilon}_{\text {ref }}^{\mathrm{T}} \vec{\sigma}_{\text {ref }} \mathrm{d} \Omega_{\text {ref }}$

$=$ the strain energy of the reference solution,

$\frac{1}{2}\left\|\vec{u}_{h}\right\|_{s}^{2}=\frac{1}{2} \int_{\Omega_{\mathrm{ref}}} \vec{\varepsilon}_{h}^{\mathrm{T}} \vec{\sigma}_{h} \mathrm{~d} \Omega_{\mathrm{ref}}$

$\approx$ the strain energy of the target solution, but it is important to note that in general $\left\|\vec{u}_{\mathrm{ref}}-\vec{u}_{h}\right\|_{s}^{2} \neq$ $\left\|\vec{u}_{\text {ref }}\right\|_{s}^{2}-\left\|\vec{u}_{h}\right\|_{s}^{2}$.

\section{References}

[1] Bathe KJ. Finite element procedures. New Jersey: Prentice Hall; 1996.

[2] Chapelle D, Bathe KJ. The finite element analysis of shells fundamentals. Berlin: Springer; 2003.

[3] Chapelle D, Bathe KJ. Fundamental considerations for the finite element analysis of shell structures. Comput Struct 1998;66:19-36, 711-712.

[4] Lee PS, Bathe KJ. On the asymptotic behavior of shell structures and the evaluation in finite element solutions. Comput Struct 2002;80:235-55.

[5] Bathe KJ, Zhang H. Finite element developments for general fluid flows with structural interactions. Int $\mathbf{J}$ Numer Meth Eng 2004;60:213-32.

[6] Bernadou M. Finite element methods for thin shell problems. New York: John Wiley \& Sons; 1996.

[7] Chapelle D, Bathe KJ. The mathematical shell model underlying general shell elements. Int J Numer Meth Eng 2000;48:289-313.

[8] Bucalem ML, Nóbrega SHS. A mixed formulation for general triangular isoparametric shell elements based on the degenerated solid approach. Comput Struct 2000;78:35-44.

[9] Lee PS, Bathe KJ. Development of MITC isotropic triangular shell finite elements. Comput Struct 2004;82:945-62.

[10] To CWS, Liu ML. Hybrid strain based three-node flat triangular shell elements. Finite Elem Anal Des 1994;17:169-203.

[11] Hiller JF, Bathe KJ. Measuring convergence of mixed finite element discretizations: an application to shell structures. Comput Struct 2002;81:639-54.

[12] Bletzinger KU, Bischoff M, Ramm E. A unified approach for shearlocking-free triangular and rectangular shell finite elements. Comput Struct 2000;75:321-34.

[13] Lee PS, Bathe KJ. Insight into finite element shell discretizations by use of the basic shell mathematical model. Comput Struct 2005;83:69-90.

[14] Bathe KJ, Iosilevich A, Chapelle D. An evaluation of the MITC shell elements. Comp Struct 2000;75:1-30.

[15] Kojic M, Bathe KJ. Inelastic analysis of solids and structures. Berlin: Springer; 2005. 